Genet. Sel. Evol. 38 (2006) 1

(C) INRA, EDP Sciences, 2005

DOI: $10.1051 / \mathrm{gse}: 2005031$

\title{
GSE MOVES TO A NEW SYSTEM OF SUBMISSION
}

Since October 10, 2005 the management of manuscripts submitted to GSE has moved to a new electronic system: Management Manuscript System or MMS. This system offers different services, which facilitate submission of manuscripts and contribute to their efficient and rapid processing. It is accessible at: http://www.edpsciences.org/gse. With this system, we expect to process your manuscripts quickly and efficiently.

In the editorial board, two new scientific editors: Hélène Hayes (INRA, Jouy-en-Josas, France) and Philippe Baret (Université catholique de Louvain, Belgium) have taken over from Francis Minvielle, after six years of active and successful participation as Scientific Editor. H. Hayes will address manuscripts in molecular biology, while P. Baret will deal with those in quantitative genetics. Didier Boichard (INRA, Jouy-en-Josas, France) will act as Editor-in-Chief. The journal thanks Michel Georges for his continuous help, as former Editor-in-Chief, to improve the quality and the international audience of GSE.

For the fourth consecutive year, the impact factor of GSE is in progression (1.65 in 2004). Most of the articles published in GSE have focused on original research in quantitative and molecular genetics of farm and experimental animals, as well as related species. Since the number of submissions has markedly increased over the last two years, GSE has slightly modified its scope by restricting it to domesticated and experimental species and leaving articles on wild species to more specialised journals. The expected outcome is that GSE will specialize on papers with a strong methodological dimension to help understand, maintain and exploit animal genetic variability. 